\title{
Parametric Uncertainty Quantification Using Polynomial Chaos Expansions Applied to a Wet Friction Clutch Model
}

\author{
Georges Tod, Wannes De Groote, Tom Lefebvre, Nele De Geeter, Bruno Depraetere, and Guillaume \\ Crevecoeur
}

\begin{abstract}
Recently, designing mechatronic systems has become more and more dependent on models that are used to predict performance in a virtual environment, and the models involved are becoming increasingly more complex multiphysical systems. Instead of spending much time modeling increasingly detailed physical models, uncertainties can be explicitly considered to model the lack of knowledge. The mismatch between real-life experiments and model simulations due to parametric uncertainties can be quantified using likelihood estimation and Monte Carlo sampling techniques for propagation. In this paper, we attempt to significantly accelerate the process using polynomial chaos expansions for propagation and a genetic algorithm to maximize likelihood. The soundness of this approach is demonstrated on a wet friction clutch system. The results show that the method has a strong potential for scalability with respect to the number of uncertain parameters.
\end{abstract}

Index Terms-Polynomial chaos expansions, system identification, uncertainty quantification, wet clutch

\section{NOMENCLATURE}

$\alpha \quad$ Constant for clutch plate geometry and dynamic friction coefficient

$\beta \quad$ Fraction of $z_{\text {contact }}$ at which $P(t)=x_{1}(t)$

$\gamma \quad$ Constant for clutch plate geometry and fluid viscosity

$\Omega(t) \quad$ Angular velocity of the input shaft

$\omega \quad$ Angular velocity of the output shaft

$\omega_{\mathrm{m}} \quad$ Motor speed

$a, k, \tau \quad$ Current bias, gain, and time delay

$a_{2}, b_{2}, c_{2}$, Oil pressure to piston position transfer function

$d_{2} \quad$ coefficients

aa Oil pressure bias in piston position computation

$f_{\text {reg }}(t) \quad$ Regularization function between torque transfer phases

$f_{s k \mathrm{TC}} \quad$ Torque converter capacity factor function

$f_{s t \mathrm{TC}} \quad$ Torque converter torque ratio function

$g_{r} \quad$ Gear ratio between the output shaft of the clutch and the load shaft

$J_{i}, T_{i c}, b_{i c}$ Input shaft inertia, Coulomb friction torque, and viscous friction coefficient

$J_{o}, T_{o c}, \quad$ Output shaft inertia, Coulomb friction

$b_{o c} \quad$ coefficient, and viscous friction coefficient

$k_{\mathrm{T}} \quad$ Gain associated with oil temperature

$P(t) \quad$ Clutch plate contact pressure

Manuscript received September 28, 2018; revised May 1, 2019.

Georges Tod, Bruno Depraetere, and Suzanne Van Poppel are with 1CoDesigns and 2Decisions at Flanders Make, Belgium (e-mail: georges.tod@flandersmake.be).

Wannes De Groote, Tom Lefebvre, Nele De Geeter, and Guillaume Crevecoeur are with 3EEMMeCS, Ghent University, and 4EEDT Decision \& Control, Flanders Make, Belgium (e-mail: wannes.degroote@ugent.be).

$\begin{array}{ll}u(t) & \text { Current } \\ x_{1}(t) & \text { Oil pressure inside the chamber } \\ x_{2}(t) & \text { Oil pressure inside the chamber time derivative } \\ x_{3}(t) & \text { State used to compute the piston position } \\ z(t) & \text { Piston position } \\ z_{\text {contact }} & \text { Maximum displacement of the piston }\end{array}$

\section{INTRODUCTION}

Recently, designing products has become more and more dependent on computational models that are used to predict performance in a virtual environment. This allows the design process to be shorter, resulting in faster design iterations that lead to more robust and reliable products at lower production costs. In addition, some trends in product design have received some interest from the industrial sector to deliver smarter and more automated products to their customers. These trends translate into the need to deliver as fast as possible models of products that can predict design performance. In this context, the models involved are usually those of mechatronic systems, which are becoming increasingly more complex multiphysical systems. In order to validate such models, these are usually compared to experimental results. However, a mismatch will always remain between real-life experiments and model simulations. If, despite the mismatch, the model can still predict trends acceptably enough, the model might still be considered to be useful [1]. If the model predictability is not high enough, there are two main approaches to improve its performance:

- Spend more time to understand the underlying physics and build a more detailed physical model.

- Represent the lack of knowledge explicitly to compensate for the model structure and parameters that cannot be easily determined.

The first approach is the most comprehensive one, but it cannot be achieved in a reasonable time when the system is too complex. The second approach provides a chance to find a balance between the modeling effort and the model usefulness. However, the representation of uncertainty in the model structure or parameters will result in uncertain predictions; the challenge is, therefore, to quantify the sufficient uncertainty that allows predicting experimental observations.

In this paper, a strong assumption is made that the model structure is known and that the uncertainty is only of a parametric nature and probabilistic. The uncertainty that is assumed to remain in a given model is due to the difficulty or impossibility of measuring some physical parameters, such as friction coefficients. Additionally, it is assumed that the uncertainty structure is fixed and only its parameters, such as 
the mean and standard deviation (for a normal distribution), need to be identified. Sampling techniques such as Monte Carlo (MC) methods [2] have been extensively used for parametric uncertainty propagation. Such an approach to propagation involves sampling values for each uncertain parameter from their uncertainty range, followed by evaluating a model using this parameter set and repeating the process many times to obtain a probability distribution of the model output. The accuracy of the result depends on the number of runs performed, and generally a large number is usually necessary. As a result, this approach is computationally expensive. More structured sampling techniques, such as Latin hypercube sampling [3], adaptive sampling [3], and importance sampling [4], have been proposed with modest computational speed improvements for complex systems. More recent works have shown that functional expansions such as polynomial chaos expansions (PCEs) [5]-[7] can lead to significant gains in terms of computational costs [6]. When dealing with complex models, the use of PCEs can be facilitated by nonintrusive PCEs [7], by allowing performing the propagation without the need to modify the model itself. In this work, the model parametric uncertainty is represented using normal distributions. In addition, a term (zero-mean normally distributed) is added at the output of the model. Other distributions could have been employed without any loss of generality of the approach. The originality of this investigation lies in the use of PCEs for the propagation of parametric uncertainties inside a maximum likelihood estimation (MLE) [8, 9] loop (see Fig. 1). The goal of this approach is to find the sufficient parametric uncertainty that a model needs to include, in order to explain different experiments [10]. To maximize the likelihood of an experiment being predicted by the model, a genetic algorithm (GA) is used to select the parameters of the chosen uncertainty structure (mean and standard deviation). As GAs are based on heuristic rules, it is not guaranteed to find a global maximum. However, this method is particularly useful for complex problems (in which developing a more detailed physical model would be very time-consuming) and for simulation models that are computationally expensive.

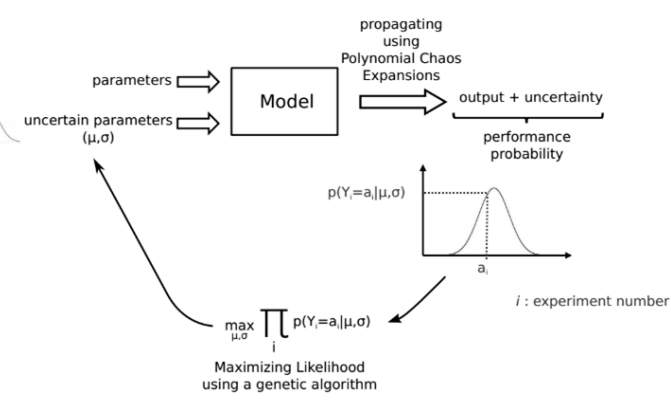

Fig. 1. The proposed approach for uncertainty quantification.

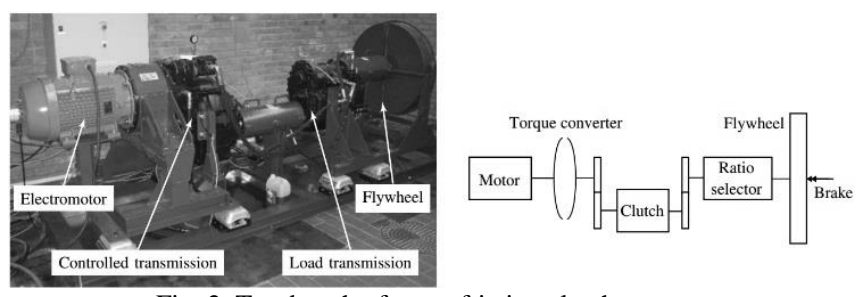

Fig. 2. Test bench of a wet friction clutch system.
As a proof of concept, this approach is applied to a wet friction clutch system (see Fig. 2). Wet friction clutches are hydraulic-mechanical devices that are used to transmit torque from their input shaft to their output shaft by means of friction. They are used in various types of automatic transmissions to selectively engage gear elements and are used in off-road vehicles and agricultural machines in which high torques are transmitted. For our investigation, a test bench was used as shown in Fig. 2.

In order to illustrate the proposed approach for uncertainty quantification, the paper starts with a section discussing the experiments that were performed and how an initial deterministic model of the physical system was derived and how it performs. The subsequent section describes the theoretical foundations of the approach and the numerical results in application to the wet friction clutch.

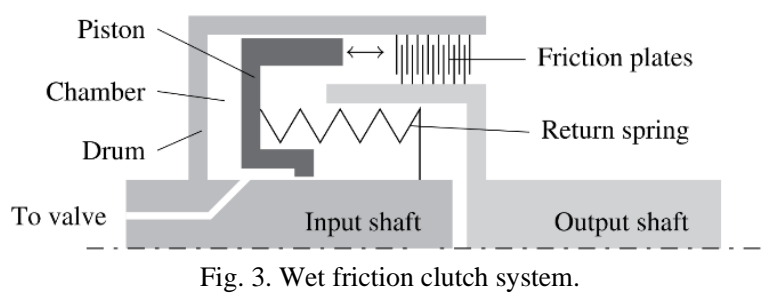

\section{WeT-CluTCH EXPERIMENTS AND MODEL}

This section describes the experiments that were performed on the test bench (Fig. 2) and how a physical model of the system was derived.

A schematic cross section of a wet clutch is shown in Fig. 3. As explained in [11], its input shaft is connected to a hollow cylinder with internal grooves, called the drum. The first set of friction plates (clutch plates) with external toothing can slide in those grooves, whereas the second set of friction plates (clutch discs) with internal toothing can slide over a grooved bus connected to the output shaft. Torque is then transferred between the shafts by pressing both sets together with a hydraulic piston, realized by sending a control signal to the servo valve in the hydraulic line to the clutch. When this happens, the clutch chamber first fills up with oil and pressure builds up until it is high enough to compress the return spring and move the piston toward the friction plates (see Fig. 4). This is called the filling phase, which ends once the piston advances far enough and presses the plates together such that torque transfer commences. At this moment, the slip phase begins and the system dynamics considerably change, yielding strongly nonlinear system behavior [12, 13]. The difference in the rotation speed between the input and the output shafts, denoted as the slip, then decreases until both shafts rotate synchronously. Good engagement is obtained when the torque transfer starts as soon as possible without introducing torque peaks, which can be realized by a short filling phase and a smooth transition into the slip phase. The pressure in the line of the clutch, the rotational speeds of the input and output shafts, and the torque of the output shaft are measured.

\section{A. Experiments}

In total, 216 experiments were performed, and the oil pressure, angular velocity, and output shaft torque were 
recorded. Between the experiments, variations of the control signal, motor speed, and inertia and friction of the load were performed (see Fig. 2). A classic key performance indicator (KPI) of interest in industry is the shifting time from neutral to the first gear in an automatic transmission (see Fig. 4). The KPIs obtained experimentally are reported in Fig. 5, and the difference between experimental and simulation results $(\Delta t)$ is shown in Fig. 5. Even though four parameters could change during the experiments, some important quantities remain incontrollable and unmeasurable on the test bench, such as the oil temperature dynamics and the piston position. The piston position is generally not measured in industrial machines, which makes the case closer to an industrial application.
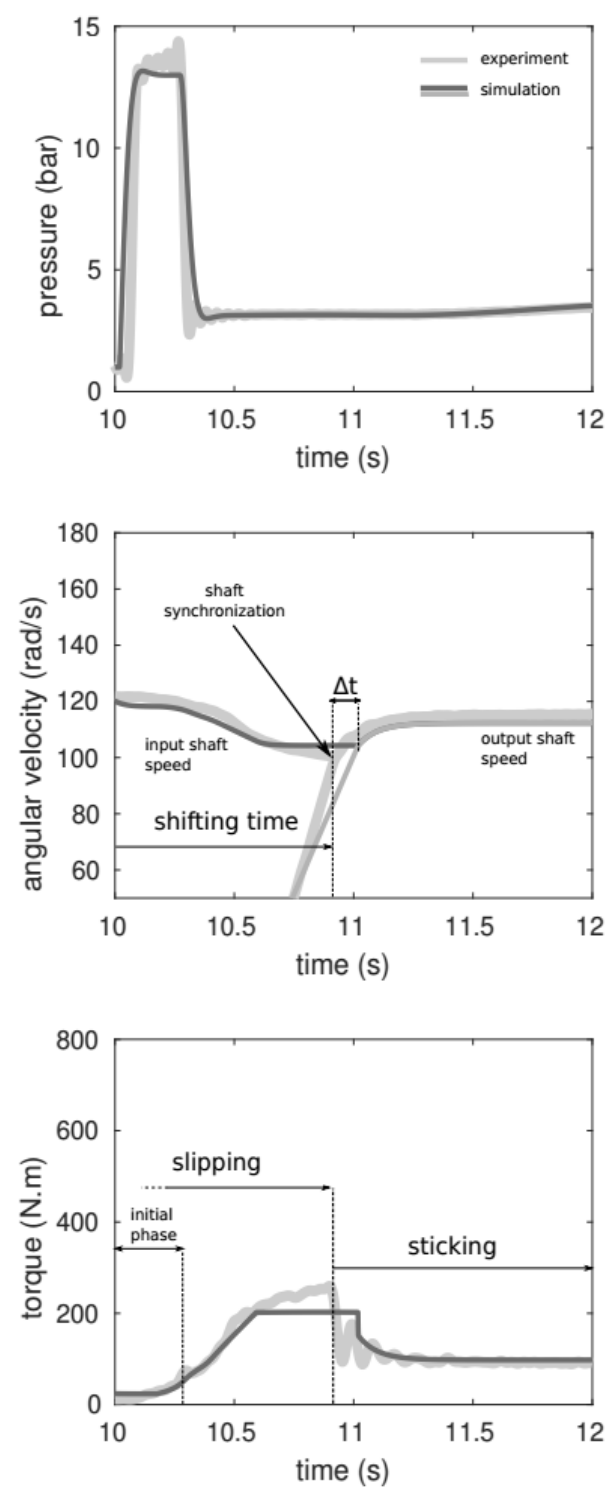

Fig. 4. Wet friction clutch system dynamics.

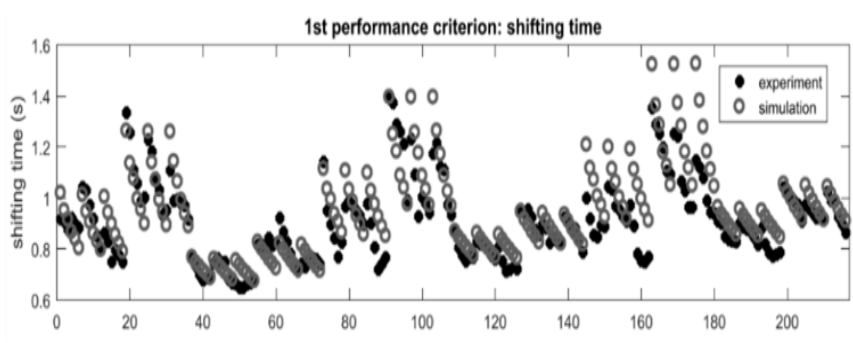

Fig. 5. Experimental versus deterministic simulation results.

\section{B. Physical Modeling}

In order to predict performance using a physical model, the test bench system was virtually decomposed into subsystems. The motor was idealized by assuming that it always delivers a predefined speed and the required torque. The torque converter is modeled using a torque ratio function, $\mathrm{f}_{\text {stTC }}\left(\frac{\omega_{\text {input }}}{\omega_{\text {output }}}\right)=\frac{\mathrm{T}_{\text {output }}}{\mathrm{T}_{\text {input }}}$, and a capacity factor function, $f_{\text {skTC }}\left(\frac{\omega_{\text {output }}}{\omega_{\text {input }}}\right)=\frac{\omega_{\text {input }}}{\sqrt{T_{\text {input }}}}$, provided by the manufacturer. The load of the system is composed of a ratio selector, a flywheel, and a brake (see Fig. 2). The ratio selector is a gearbox that allows varying the inertia, whereas the brake allows varying the friction load experienced by the clutch.

In addition, a state-space model identified on the basis of previous work [14] was used to represent the dynamics from the current input signal to the piston position in the chamber:

$$
\left\{\begin{array}{c}
\dot{x_{1}=x_{2}} \\
\dot{x_{2}}=-\frac{6}{\tau^{2}} \cdot x_{1}-\frac{4}{\tau} \cdot x_{2}-\frac{6 \cdot a \cdot k}{\tau^{2}}+\frac{6 \cdot k}{\tau^{2}} \cdot u-\frac{2 \cdot k}{\tau} \cdot \dot{u} \\
\dot{x_{3}}=\frac{b_{2}}{c_{2}} \cdot x_{1}+\frac{a_{2}}{c_{2}} \cdot x_{2}-\frac{d_{2}}{c_{2}} \cdot x_{3}-\frac{a a \cdot b_{2}}{c_{2}} \\
z=\min \left(z_{\text {contact }} \max \left(0, k_{T} \cdot x_{3}\right)\right) \\
P=\max \left(0, \frac{\frac{z}{z_{\text {contact }}}-\beta}{1-\beta}\right) \cdot 10^{5} \cdot x_{1}
\end{array}\right.
$$

The wet friction clutch model is decomposed according to the three torque phases shown in Fig. 4. The initial torque phase is the phase at which the friction plates are not yet in contact but some torque is transferred between the input and output shafts when there is oil between them. This phase is modeled according to the planar Couette flow assumption and leads to the following equation:

$$
J_{o} \cdot \frac{d \omega}{d t}=\frac{\gamma}{Z} \cdot(\omega-\Omega)
$$

where $J_{o}$ and $\omega$ are the inertia and speed of the output shaft, respectively, $z$ is the distance between the friction plates, $\Omega$ is the speed of the input shaft, and $\gamma$ is a constant that captures the geometry of the plates and the fluid viscosity. The slipping phase is the one at which the plates come into contact. This is modeled as it would be assumed for a dry friction clutch:

$$
\left\{\begin{array}{c}
J_{i} \cdot \dot{\Omega}=-b_{i v} \cdot \Omega-T_{i c}-f_{\text {reg }} \cdot \alpha \cdot P \\
+\omega_{m}^{2} \cdot \frac{f_{\text {stTC }}\left(\frac{\Omega}{\omega_{m}}\right)}{f_{\text {skTC }}\left(\frac{\Omega}{\omega_{m}}\right)^{2}}-\left(1-f_{\text {reg }}\right) \cdot \frac{\gamma}{z_{\text {contact }}-z} \cdot\left(\omega-\frac{1}{g_{r}} \Omega\right) \\
J_{o} \cdot \dot{\omega}=-b_{\text {ov }} \cdot \omega-T_{o c} \\
+f_{\text {reg }} \cdot g_{r} \cdot \alpha \cdot P+\left(1-f_{\text {reg }}\right) \cdot \frac{g_{r} \cdot \gamma}{z_{\text {contact }}-z} \cdot\left(\omega-\frac{1}{g_{r}} \Omega\right)
\end{array}\right.
$$

In the sticking phase, the plates are in contact; the input and output shaft speeds become equal; and as soon as the required torque is not too high, the transferred torque becomes independent of the oil pressure: 


$$
\begin{aligned}
& J_{i}+\frac{J_{o}}{g_{r}^{2}} \cdot \dot{\Omega}=-b_{i v} \cdot \Omega-T_{i c} \\
& +\omega_{m}^{2} \cdot \frac{f_{\text {stTC }}\left(\frac{\Omega}{\omega_{m}}\right)}{f_{\text {skTC }}\left(\frac{\Omega}{\omega_{m}}\right)^{2}}-\frac{1}{g_{r}^{2}} b_{o v} \cdot \Omega-\frac{1}{g_{r}} \cdot T_{o c}
\end{aligned}
$$

The equations are implemented in MATLAB and solved using a solver for nonstiff differential equations (ode45).

\section{Experimental versus Deterministic Simulation Results}

The model contains parameters (e.g., viscous friction coefficients and Coulomb friction coefficients) that were identified using specific experiments in steady state. Inertias were estimated on the basis of known masses, materials, and geometrical approximations. Other parameters, such as current bias, gain and delay, oil temperature, pressure bias for position computation, clutch friction coefficient, and initial piston position, cannot be measured and are considered uncertain. As a first approximation, these parameters are fitted by nonlinear least squares using the experimental results. A comparison between the experimental and the simulation results can be found in Fig. 5. The difference between the experimental and simulation results $(\Delta t)$ is shown in Fig. 5. As mentioned in the previous paragraphs, the oil temperature can hardly be measured and there is no piston position sensor on our setup. The lack of knowledge of these dynamics is a source of uncertainty that the method proposes to quantify. In order to reduce the influence of oil temperature dynamics, all experiments were performed after warming up the machine, which guarantees some identical initial conditions in terms of temperature. It is assumed that the uncertainty of the model is of only a parametric nature. Future work should investigate how to include model structure uncertainties as well.

\section{PARAMETRIC UNCERTAINTY QUANTIFICATION}

Parametric uncertainty quantification is performed according to the approach shown in Fig. 1. In order to verify this approach, the results will be compared to a densely sampled quasi-MC (for propagation) and exhaustive grid search (which maximizes likelihood), which we will refer to as "classic" throughout the rest of this paper. This classic approach allows us to brute-force the solution and can be very effective when the number of uncertain parameters is not too high or the computational cost of evaluating the model is very low. The proposed approach in this paper allows overcoming those two limitations. The main originality is that, for such class of problems, PCEs are applied for the propagation of parametric probabilistic uncertainties. This section presents some theory of PCEs and the validation of the proposed approach on the wet friction clutch model that was described in the previous section.

\section{A. Propagation Using PCEs}

As determined by the architecture in Fig. 1, we need an efficient means to quantify the probability that the performance $\left(y_{i}\right)$ of experiment $i$, as obtained via simulation, takes the same value as that for the experimentally measured performance $\left(a_{i}\right)$ and that for a specified input uncertainty. Mathematically, we need to evaluate the conditional output probability density function, $p_{Y_{i}}\left(a_{i} \mid \beta\right)$, which is a parameter set that fully determines the input uncertainty.

To that end, we will engage the PCE framework [15]. This framework offers an efficient and accurate tool for quantifying and propagating uncertainties in a nonlinear context and is computationally superior to brute-force MC approaches. The propagation of input uncertainties to the output space is realized by modeling an explicit forward deterministic model through polynomial series expansion as a function of random parameters. When the polynomial basis functions are chosen to be orthonormal with respect to the input probability distribution, optimal approximation conditions are established to propagate uncertainties to the output space for that specific input distribution [15]. Numerical details are provided next.

According to the framework, any sufficiently smooth forward model $y(\cdot)$ can be represented as infinite polynomial series expansion [16].

$$
\mathrm{y}(x)=\sum_{i=0}^{\infty} c_{i} \phi_{i}(x) \rightarrow y_{(d)}(x)=\sum_{i=0}^{n_{p}} c_{i} \phi_{i}(x)
$$

Here, $c_{i}$ represents the polynomial coefficients and $\phi_{i}(\cdot)$ is the range of polynomial basis functions that can be used to write any polynomial. For practical purposes, we consider the $n_{d}$ th-order approximation, $y_{(d)}(\cdot)$, that is obtained by omitting any higher-order polynomials from the series expansion and, thus, omitting any polynomial basis function $\phi_{i}(\cdot)$ of order $>n_{d}$. When the basis is ordered properly, this corresponds with truncating the series after the $n_{p}$ th term. The latter value is determined by the polynomial order $\left(n_{d}\right)$ and the input dimension $\left(n_{x}\right)$ as $n_{p}=\frac{\left(n_{d}+n_{x}\right) !}{n_{d} ! n_{x} !}$. The polynomial order required to represent a given forward model accurately is determined by the model's nonlinearity and input dimension. Exact error bounds as a function of the polynomial order are given in [17]. The error vanishes for $d \rightarrow \infty$.

Now, consider that the input parameter $x$ corresponds to a stochastic variable $X$. We are interested in quantifying the stochastic behavior of the output variable $Y=y(X)$. As mentioned, $y_{(d)}(\cdot)$ is suited optimally to quantify uncertainty propagation to the output space when the polynomials are orthonormal with respect to the probability distribution of $X$. A polynomial basis is orthonormal with respect to the arbitrary probability distribution $p_{X}(\cdot)$ when the following condition holds (here, $\mathcal{X}$ represents the value set of the input uncertainty):

$$
\left\langle\phi_{i}, \phi_{j}\right\rangle=\int_{x} \phi_{i}(x) \phi_{j}(x) p_{X}(x) \mathrm{d} x .
$$

Several standard probability distributions are associated with known univariate orthonormal polynomial bases by the Wiener-Askey scheme [15] (see Table I). A multivariate orthonormal basis is easily constructed from a set of univariate orthonormal bases under the sole assumption that the stochastic input variables are independent. How to construct such a basis is detailed in the Appendix.

In this work, two random input variables are considered, 
and it is assumed that both are normally distributed. Hence, we can express these two input variables as a linear transformation of the standard normal random variables $\left(\theta_{1}\right.$ and $\left.\theta_{2}\right)$. According to the Wiener-Askey scheme, the multivariate Hermite polynomials $\left(H_{i}\right)$ are optimal for constructing the polynomial series (see the Appendix). Notice that the forward model then becomes a function of the standard normal random variable $(\theta)$ invoking the transformation $x(\theta \mid \beta)$ that is fully determined by the parameter $\beta=\left(\mu_{1}, \mu_{2}, \sigma_{11}, \sigma_{22}, \sigma_{12}\right){ }^{1}$

$$
\mathrm{x}(\theta \mid \beta)=\left[\begin{array}{l}
x_{1} \\
x_{2}
\end{array}\right]=\left[\begin{array}{l}
\mu_{1} \\
\mu_{2}
\end{array}\right]+\left[\begin{array}{ll}
\sigma_{11} & \sigma_{12} \\
\sigma_{21} & \sigma_{22}
\end{array}\right]\left[\begin{array}{l}
\theta_{1} \\
\theta_{2}
\end{array}\right] ; \theta_{1}, \theta_{2} \sim \mathcal{N}(0,1) \text {. }
$$

Coefficients $c_{i}(\beta)$ as a function of the transformation parameters are calculated by projecting the forward model on the polynomial space exploiting the inner product definition. Notice that, indeed, all other terms vanish because of the orthonormality of the multivariate Hermite polynomials $H_{i}(\cdot)$ with respect to the multivariate normal distribution $\varphi(\cdot)$ (see the Appendix for details).

$$
\begin{aligned}
c_{i}(\beta)=\left\langle y(x(\cdot \mid \beta)), H_{i}\right\rangle \quad & =\int_{\Theta_{\Theta}} y(x(\theta \mid \beta)) H_{i}(\theta) \varphi(\theta) \mathrm{d} \theta \\
& \approx \sum_{j=1}^{n_{q}} y\left(x\left(\theta_{j} \mid \beta\right)\right) H_{i}\left(\theta_{j}\right) w_{j}
\end{aligned}
$$

In this work, quadrature rules are used to approximate the resulting integral. A quadrature rule is determined by a number of collocation points $\left(\theta_{j}\right)$ and collocation weights $\left(w_{j}\right)$. A unique optimal quadrature rule is associated with each polynomial basis covered by the Wiener-Askey scheme [17]. For a one-dimensional input, a quadrature set of $n_{q}$ points is exact for polynomials up to degree $2 n_{q}-1$. Hence, the quadrature order determines the number of coefficients that can be retrieved correctly and, therefore, the accuracy of the polynomial approximation. For a multivariate input, full tensor products can be used. We need to emphasize that the characterization of the expansion in Eq. (5) still requires performing a total of $n_{q}$ forward model evaluations. In general, however, the number of collocation points required to optimally approximate the forward model is far less than the number of MC samples required to accurately quantify the output uncertainty, justifying the computational efficiency of PCE over MC.

In conclusion, recall that we are interested in the conditional output density function with respect to the transformation parameters $(\beta)$. In order to evaluate the probability density function of the output variable, a number of $\mathrm{MC}$ simulations can be performed on the polynomial in Eq (5) using the coefficients obtained by Eq. (6), hence omitting any further direct evaluations of the forward model.

\begin{tabular}{ccc}
\multicolumn{3}{c}{ TABLE I: WIENER-ASKEY POLYNOMIAL CHAOS } \\
Distribution & Polynomials & Support \\
\hline Gaussian & Hermite & {$[-\infty, \infty]$} \\
Gamma & Laguerre & {$[0, \infty]$} \\
Beta & Jacobi & {$[-1,1]$} \\
Uniform & Legendre & {$[-1,1]$} \\
\hline \hline
\end{tabular}

\footnotetext{
${ }^{1}$ When neglecting the input correlation, we can disregard $\sigma_{12}$ by definition.
}

\section{B. Application and Numerical Results}

In order to validate the proposed approach (see Fig. 1), it is applied to the wet friction clutch model that was presented in Section 2. As previously stated, the model contains seven uncertain parameters; however, as a first step, only two uncertain parameters are considered: pressure bias and initial piston position. To vary those two uncertain parameters, the nominal pressure bias is multiplied by $x_{1}$ and the nominal initial piston position is multiplied by $x_{2}$. In order to avoid the cost of repeating computations of the model several times, the model performance (shifting time from neutral to the first gear) is stored in a lookup table for $50 \times 50$ variations of $x_{1} \times x_{2}$ between 0.5 and 1.5 for the 216 experimental conditions. 540k simulations were run on a supercomputer and stored in a lookup table that replaces the model block in the approach shown in Fig. 1. Future work should also investigate the use of surrogate models to replace, when relevant, this step of the approach.

It is assumed that the two uncertain parameters are normally distributed; however, other distributions could have been chosen without any loss of generality; the impact on the PCE would be on the kind of polynomials to be used [5].
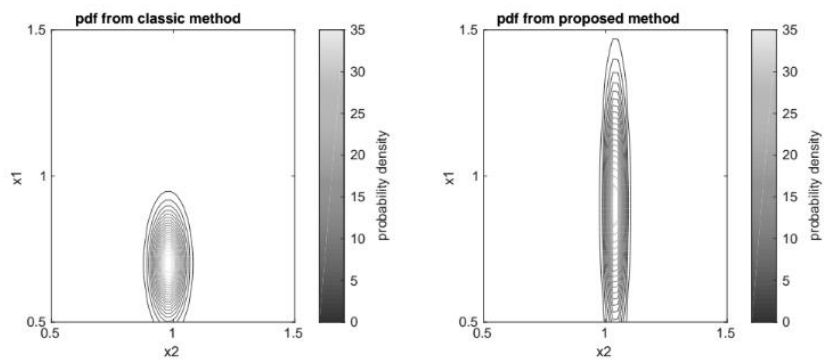

Fig. 6. Quantified uncertainty on inputs.

For a given input multivariate normal distribution of $x_{i}$, the propagation through the model and the addition of an additive output uncertainty term (zero-mean Gaussian) allows obtaining a performance probability distribution. Covariance terms were removed as they appeared to have no significant effect in this case. The likelihood of the given uncertainty $\left(\mu_{x_{1}}, \mu_{x_{2}}, \sigma_{x_{1}}, \sigma_{x_{2}}, \sigma_{\text {output }}\right)$ is then computed as the product of the probability of the simulation model to be the experimentally measured performance $\left(\Delta t_{i}\right.$ from the data) for the $i$ th experimental scenario:

$$
L(\mu, \sigma)=\prod_{i}^{216} p\left(Y_{i}=\Delta t_{i} \mid \mu, \sigma\right) .
$$

The higher the value of $L(\mu, \sigma)$ is, the more likely the simulation results are to represent the data obtained experimentally. In the classic approach, the maximization of $L$ was found by evaluating the likelihood of a very large number of combinations of means and variances. The maximum decimal log-likelihood is -269 and is plotted in Fig. 6. For a given uncertainty structure, this is the best result that can be found, and it is used as a benchmark of the proposed approach. Figure 6 shows the region of $x_{i}$ 's that best fits the data. As the $x_{2}$ 's mean is close to 1 , the nominal parameter appears to be an acceptable approximation. The probability density functions for all experiments are 
presented in Fig. 7, including the experimental results (black dots).

In comparison, the proposed approach provides an approximation of the previous solution. The maximum decimal log-likelihood was found to be -273 . An important downside of this approach is that it cannot achieve the exact same performance as that of the brute-force approach. This is due to the fact that PCEs introduce a model approximation and that a GA is used to replace the grid search, which cannot guarantee that the solution is a global optimum. However, the so-called classic brute-force approach is not scalable with the number of uncertain parameters because of its computational cost.

For the proposed approach, the grid search of uncertain inputs is replaced by a GA that dramatically reduces the number of necessary model evaluations. In addition, PCEs accelerate the propagation of uncertain parameters and lead to an interesting solution (see Fig. 6).

TABLE II: COMPUTATIONAL COST OF EACH METHOD PERFORMED ON A DESKTOP PC $(8 \times$ CORE I7-6900K, 128 GB OF RAM $)$

\begin{tabular}{cc} 
Method & Computational cost \\
\hline Classic: Quasi-MC + grid search & $\approx 277 \mathrm{~h}$ \\
Proposed: PCEs + GA & $\approx 1.65 \mathrm{~h}$ \\
\hline \hline
\end{tabular}

Owing to the low cost of the computation (see Table II), scalability is a great potential for this method. In future works, the accuracy of the result (Fig. 6) could be improved by refining the search method after this initial solution.
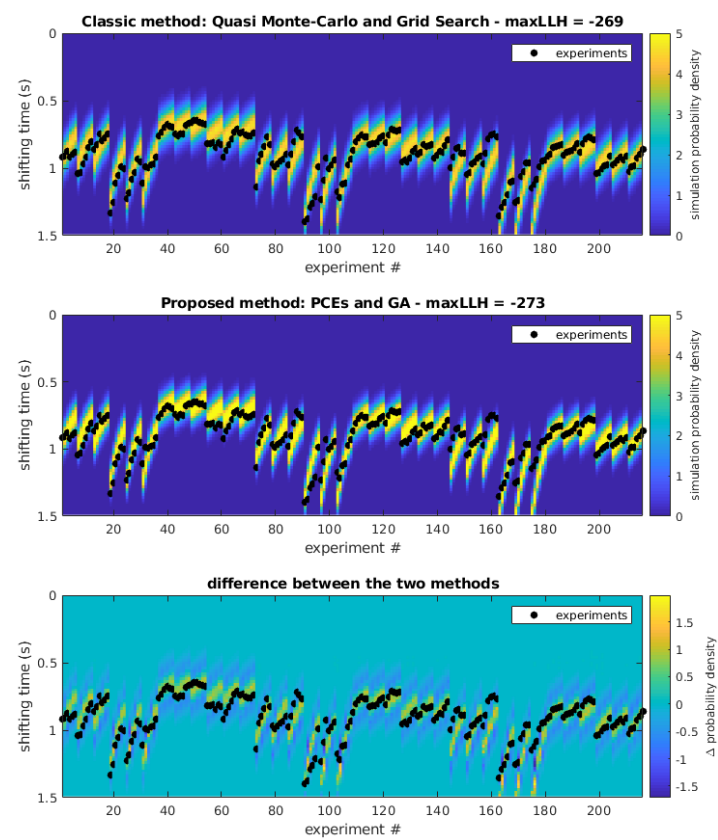

Fig. 7. Simulation performance probability versus measured performance.

\section{CONCLUSIONS AND FUTURE WORK}

In this paper, an approach using PCEs for propagation and a GA for the selection of uncertainty structure parameters was presented to quantify the sufficient parametric uncertainty that a given model should include in order to explain experimental results. It was then illustrated by applying it to the complex problem of predicting the shifting time of an automatic wet friction clutch system. The method was then validated by comparing the obtained solution with the one obtained by brute-forcing the problem. The results showed that this method provides an approximation of the solution at a very interesting computational cost. The proposed approach has, therefore, a great potential of scalability with respect to the number of uncertain parameters.

Future works should investigate the inclusion of not only parametric but also model structure uncertainties.

\section{APPENDIX}

Hermite Polynomials and Multivariate Bases

Univariate Hermite polynomials, $H_{i}(\cdot)$, are orthogonal with respect to the univariate standard normal distribution, $\mathcal{N}(0,1)$, in which the probability density function is given by $\varphi(\theta)=\sqrt{2 \pi^{-1}} e^{\frac{-\theta^{2}}{2}}$. In the main text, we mentioned that the Hermite polynomials are orthonormal, meaning that the inner product not only vanishes when applied on different Hermite polynomials but also normalizes when applied on the same Hermite polynomials. Note that, within a normalization factor, both definitions are equal; hence, we opted merely to mention the normalized version so as to ease the readability of the section. It follows that

$$
\left\langle H_{i}, H_{j}\right\rangle=\int_{-\infty}^{\infty} H_{i}(\theta) H_{j}(\theta) \frac{1}{\sqrt{2 \pi}} e^{\frac{-\theta^{2}}{2} \mathrm{~d}} \theta=\delta_{i j}
$$

In the univariate case, a basis $H_{1}^{n_{d}}$ for the univariate polynomial space of at most degree $n_{d}$ is spanned by the first $n_{d}+1$ Hermite polynomials, $\left\{H_{0}, \ldots, H_{n_{d}}\right\}$. By construction, this basis is orthogonal with respect to the univariate standard normal distribution. A basis $H_{n_{\theta}}^{n_{d}}$ for the $n_{\theta}$-variate polynomial space of at most degree $n_{d}$ can be generated from the $n_{\theta}$ univariate bases $H_{1}^{n_{d}}$. The basis vectors are defined as $H_{|i| \leq n_{d}}=\prod_{k=1}^{n_{\theta}} H_{i_{k}}\left(\theta_{k}\right)$, with multi-index $i=\left(i_{1}, \cdots, i_{n_{\theta}}\right)$, where $|i|=\sum_{k=1}^{n_{\theta}} i_{k}$. For notational convenience, we further exploit the bijection that exists between the multi-indices $i$ that satisfy $|i| \leq n_{d}$ and the integer index $i$ taking values in $\left(1, \ldots, \frac{\left(n_{\theta}+n_{d}\right) !}{n_{\theta} ! n_{d} !}\right)$. Notice that this basis is indeed orthogonal with respect to the multivariate standard normal distribution, defined as $\varphi(\theta)=\prod_{k=1}^{n_{\theta}} \varphi\left(\theta_{k}\right)$.

$$
\left\langle H_{i}, H_{j}\right\rangle=\prod_{k=1}^{n_{\theta}} \int_{-\infty}^{\infty} H_{i_{k}}(\theta) H_{j_{k}}(\theta) \varphi\left(\theta_{k}\right) \mathrm{d} \theta_{k}=\prod_{k=1}^{n_{\theta}} \delta_{i_{k} j_{k}}=\delta_{i j}
$$

\section{ACKNOWLEDGMENT}

Some of the computational resources and services used in this work were provided by VSC (Flemish Supercomputer Center), funded by the Research Foundation-Flanders (FWO) and the Flemish Government, Department of EWI. This study was performed within the framework of the EVIT ICON project and was supported by Flanders Make, a strategic research center for the manufacturing industry. 


\section{REFERENCES}

[1] A. Saltelli, M. Ratto, T. Andres, F. Campolongo, J. Cariboni, D. Gatelli, F. Campolongo, J. Cariboni, D. Gatelli, and M. Saisana, Global Sensitivity Analysis: The Primer, John Wiley \& Sons, 2008.

[2] M. H. Kalos and P. A. Whitlock, Monte Carlo Methods, Wiley \& Sons, New York, USA, 1986.

[3] J. C. Helton and F. J. Davis, Latin Hypercube Sampling and the Propagation of Uncertainty in Analyses of Complex Systems, Reliability Engineering \& System Safety, 2003.

[4] R. E. Melchers, Importance Sampling in Structural Systems, Structural Safety, 1989.

[5] A. O'Hagan, Polynomial Chaos: A Tutorial and Critique from a Statistician's Perspective, SIAM/ASA J. Uncertainty Quantification, 2013.

[6] G. Kewlani, J. Crawford, and K. Iagnemma, "A polynomial chaos approach to the analysis of vehicle dynamics under uncertainty," Vehicle System Dynamics, 2012.

[7] S. Hosder, R. W. Walters, and M. Balch, Point-collocation Nonintrusive Polynomial Chaos Method for Stochastic Computational Fluid Dynamics, AIAA journal, 2010.

[8] P. J. Bickel and A. D. Kjell, Mathematical Statistics; Basic Ideas and Selected Topics, Holden-Day, 1977.

[9] L. Ljung, "System identification," Signal Analysis and Prediction, pp. 163-173, Birkhäuser, Boston, MA, 1998.

[10] K. Berx, E. Hostens, W. Driesen, and G. Pinte, "Identification and propagation of parametric uncertainty of a hydrostatic drive train model," International Conference on Noise and Vibration Engineering, 2014.

[11] B. Depraetere, G. Pinte, W. Symens, and J. Swevers, "A two-level iterative learning control scheme for the engagement of wet clutches," Mechatronics, 2011.

[12] A. P. Ompusunggu, P. Sas, and H. V. Brussel, "Modeling and simulation of the engagement dynamics of a wet friction clutch system subjected to degradation: An application to condition monitoring and prognostics," Mechatronics, 2013.

[13] S. Iqbal, F. Al-Bender, A. P. Ompusunggu, B. Pluymers, and W. Desmet, "Modeling and analysis of wet friction clutch engagement dynamics," Mechanical Systems and Signal Processing, 2015.

[14] W. D. Widanage, J. Stoev, A. V. Mulders, J. Schoukens, and G. Pinte, Nonlinear System-Identification of the Filling Phase of a Wet-Clutch System, Control Engineering Practice, 2011.

[15] D. Xiu and G. Karniadakis, "The wiener-askey polynomial chaos for stochastic differential equations," SIAM Journal on Scientific Computing, 2002.

[16] K. K. K. Kim, D. E. Shen, Z. K. Nagy, R. D. Braatz, "Wiener's polynomial chaos for the analysis and control of nonlinear dynamical systems with probabilistic uncertainties," IEEE Control Systems, vol. 33, no. 5, pp. 58-67, 2013.

[17] D. Xiu, "Efficient collocational approach for parametric uncertainty analysis," Commun. Comput. Phys, vol. 2, no. 2, pp. 293-309, 2007.

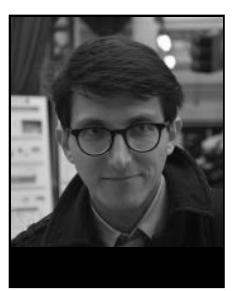

Georges Tod received both of his M.S. and Ph.D. degrees in mechanical engineering from Arts et Métiers ParisTech, Paris, France, in 2010 and 2015, respectively. He worked at Airbus Helicopters as a Rotor Analysis \& Design Engineer, as a Research Assistant (focusing on helicopter dynamics), and as a Visiting Researcher at the Faculty of Aerospace Engineering of Delft University of Technology, the Netherlands. Since 2018, he has been working as a Research Engineer at Flanders Make, Belgium, within the CoDesigns core lab. His research interests include the modeling and simulation of complex dynamic systems and optimization algorithms to improve their design.

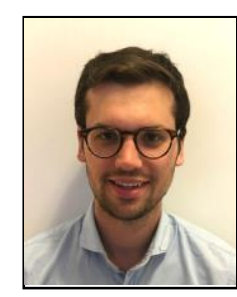

Wannes De Groote was born in Knokke-Heist, Belgium, in 1993. He received his M.S. degree in electrical and mechanical engineering from Ghent University, Ghent, Belgium, in 2017. In 2015, he enrolled in an exchange program at Grenoble INP, Grenoble, France. In August 2017, he joined the Department of Electrical Energy, Metals, Mechanical Constructions and Systems, Ghent University, as a Ph.D. Student. He received a personal grant, Strategic Basic Research (SB), from Research Foundation-
Flanders (FWO) in 2019. His research interests mainly include the simulation and modeling of dynamic systems by combining physical and data-driven approaches. Mr. De Groote is an affiliate member of Flanders Make, a strategic research center for the manufacturing industry in Flanders, Belgium.

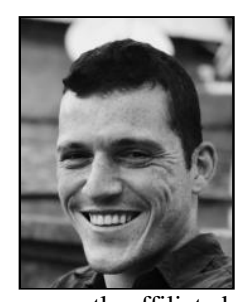

Tom Lefebvre received his M.S. degree in electrical and mechanical engineering from Ghent University, Ghent, Belgium, in 2015, where he is currently pursuing his Ph.D. degree with the Department of Electrical Energy, Metals, Mechanical Constructions and Systems. His current research interests include optimal control theory, stochastic optimal control, and stochastic evolutionary optimization strategies. Lefebvre is currently affiliated with Flanders Make, a strategic research center for the manufacturing industry in Flanders, Belgium.

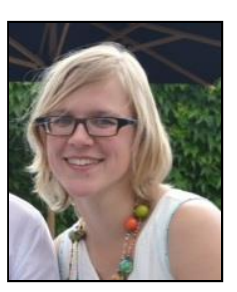

Nele De Geeter was born in Belgium in 1986. In 2009, she obtained her M.S. degree in electromechanical engineering at Ghent University, Ghent, Belgium. She then joined the Department of Electrical Energy, Metals, Mechanical Constructions and Systems, Ghent University, where she obtained in 2015 her D.Eng. degree. Her research interests are numerical methods for bioelectromagnetism and modeling uncertainties using polynomial chaos. Since 2017, she has been working as a Full-Time Educational Supervisor at the same department at Ghent University.

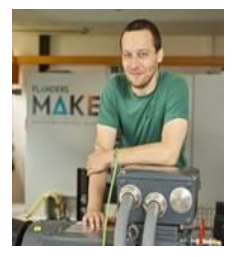

Bruno Depraetere received his M.S. degree in electromechanical engineering and his $\mathrm{Ph} . \mathrm{D}$. degree in engineering science from KU Leuven, Belgium, in 2008 and 2012, respectively. He is now a Research Engineer at Flanders Make, the Flemish Strategic Research Center for the Manufacturing Industry, Leuven, Belgium, performing precompetitive industry-driven research on advanced control and its industrial applications.

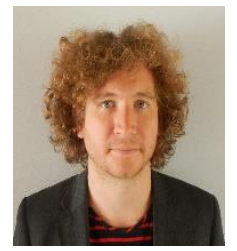

Guillaume Crevecoeur has been an Associate Professor at Ghent University since 2014. He received his M.S. and Ph.D. degrees in engineering physics from Ghent University in 2004 and 2009, respectively. In 2004, he joined the Department of Electrical Energy, Metals, Mechanical Constructions and Systems as a doctoral student working on optimization and system identification. In 2009, he became a Postdoctoral Fellow at the Research FoundationFlanders (FWO). In the winter of 2011, he worked as a Visiting Researcher at Technical University Ilmenau and Physikalisch-Technische Bundesanstalt, Berlin, Germany. He is the leader of an interdisciplinary research team working on modeling, optimization, and control of dynamical systems with applications on electromechanical systems. Furthermore, he is the leader of the Flanders Make @ UGent-EEDT core lab within the competence cluster Decision and Control. He has carried out foundational work on system identification and nonlinear optimal control, as well as their combination with artificial intelligence. He is currently carrying out strategic research on artificial intelligence to incorporate algorithms and methodologies into physical systems in the real world. 